\title{
Cross-layer routing and scheduling for IEEE 802.16 mesh network
}

\begin{abstract}
In the last few years, demand for high-speed internet access and multimedia service has increased greatly. The IEEE 802.16 standard defines the wireless broadband access technology called WiMAX (Worldwide Interoperability Microwave Access) aims to provide broadband wireless last-mile access, easy deployment, high speed data rate for large spanning area. In this paper, we propose an Energy/bit Minimization routing and centralized scheduling (EbM-CS) based algorithm to multi-transceiver in WiMax mesh network (WMN), which introduces the cross-layer concept between the MAC and network layers. The results show that our algorithm has improved the system performance in the aspect of system throughput.
\end{abstract}

Keyword: WiMAX; EbM routing; Centralized scheduling; Throughput; Multi-transceiver; Cross layer 